\title{
Perfil empreendedor dos estudantes de Odontologia da Universidade Federal de Santa Catarina
}

Gustavo Baur*; Murillo Barreto Cardoso*; Vinícius Spiger**; Cláudio José Amante***

* Cirurgião-dentista graduado pela Universidade Federal de Santa Catarina.

** Cirurgião-dentista, aluno de Mestrado no Programa de Pós-Graduação em Odontologia da Universidade Federal de Santa Catarina.

*** Doutor em Engenharia de Produção, Professor do Departamento de Odontologia na Universidade Federal de Santa Catarina.

\section{RESUMO}

Este estudo objetivou verificar o perfil empreendedor entre os estudantes do Curso de Graduação em Odontologia da Universidade Federal de Santa Catarina, através de uma pesquisa descritiva do tipo transversal, realizada em abril de 2015, com alunos regularmente matriculados que já haviam concluído a disciplina Clínica I. A coleta de dados foi realizada em sala de aula, por meio do Questionário Sociodemográfico, do Questionário de Classificação Econômica do Brasil e do Teste do Perfil Empreendedor, instrumento que avalia, através de um sistema de quatro pontos, o perfil empreendedor, classificando-o em alto, médio ou baixo. Os dados foram então tabulados e submetidos à análise estatística descritiva. Entre os 94 participantes da pesquisa, o sexo feminino foi predominante $(73,34 \%)$. As classes econômicas mais frequentes foram a Classe A $(50,00 \%)$ e a Classe B2 $(22,34 \%)$, sem nenhum participante da Classe $\mathrm{D}$ ou $\mathrm{E}$. Observou-se predominância do perfil empreendedor médio $(58,51 \%)$, em relação ao alto $(41,49 \%)$. Nenhum estudante apresentou perfil baixo. Entre os estudantes de perfil empreendedor alto, $76,92 \%$ eram do sexo feminino. A classe econômica $\mathrm{C} 2$, apenas composta por dois indivíduos, apresentou maior frequência como perfil empreendedor alto $(50 \%)$, seguida das classes A1 $(46,81 \%)$ e C1 $(46,15 \%)$. Assim, essa pesquisa possibilitou identificar o perfil empreendedor entre os estudantes de odontologia da Universidade Federal de Santa Catarina, onde embora não exista um perfil empreendedor baixo, existe a necessidade de estimular o perfil empreendedor alto. Há também um destaque para a tendência empreendedora no sexo feminino.

Descritores: Estudantes de Odontologia. Inovação. Empreendedorismo. Organização e Administração.

\section{INTRODUÇÃO}

A Odontologia vive, atualmente, um momento de grande concorrência em seu mercado de trabalho, o qual é anualmente abastecido por uma significativa quantidade de novos profissionais, provenientes de aproximadamente 216 cursos existentes no país ${ }^{1}$. 
Há também um notável aumento na mobilidade e no comércio nacional, resultando em uma crescente necessidade pela busca de visões mais amplas, através de indivíduos capacitados para identificar ameaças e oportunidades, com um verdadeiro perfil empreendedor ${ }^{2,3}$.

O recém-formado encara um mercado de trabalho que exige tanto a atualização de conhecimentos técnico-científicos quanto a de conhecimentos administrativos e econômicos ${ }^{4}$.

As opções de carreira para o graduando em Odontologia podem ser resumidas em três grandes áreas: a contratação, que se dá por serviço público ou privado; a atuação como profissional liberal, com responsabilidades pela gestão do próprio negócio; ou ainda a carreira acadêmica ${ }^{5}$.

Independente do caminho a ser percorrido, pelas mudanças no mercado, a qualidade do serviço prestado deixa de depender somente do bom exercício da Odontologia, e passa também a englobar as capacidades de gestão, de infraestrutura, de inovação, de atendimento ao cliente, entre outras ${ }^{4}$. Assim, o sucesso profissional do cirurgião-dentista exige uma ampla quantidade de habilidades ${ }^{6}$.

Entretanto, esses conhecimentos e habilidades não surgem de maneira espontânea no profissional. A participação das universidades em formações mais abrangentes para os alunos de Odontologia, é fundamental, dessa maneira estimulando o espírito empreendedor ${ }^{7,8}$.

Nesse sentido, as Diretrizes Curriculares Nacionais que regem o ensino a nível de graduação em Odontologia almejam à formação de um cirurgião-dentista generalista, que, para além das necessárias capacidades técnico-científicas, possua também competências em gestão e liderança ${ }^{9}$.

Através da administração e do gerenciamento, o profissional é capaz de desenvolver a aptidão para os processos de tomada de decisão e de administração das forças de trabalho e de informação. A liderança é necessária para possibilitar o adequado trabalho nas equipes multiprofissionais. $\mathrm{O}$ ensino deve, portanto, ser capaz de formar cirurgiões-dentistas que liderem equipes, tendo sempre em vista o bem-estar da população, com a responsabilidade, a empatia e a habilidade para estas competências, através de conhecimentos como a contabilidade, a administração e a logística ${ }^{9,10}$.

Faz-se então necessária uma linha pedagógica voltada para a prática, capaz de fornecer o respaldo de aspectos como habilidades e competências de atividades criativas, inovação, tomada de decisões, entre outras questões relacionadas ao empreendedorismo ${ }^{8}$.

Dada a importância das habilidades empreendedoras para a formação do cirurgião-dentista, este estudo teve como objetivo identificar o perfil empreendedor entre estudantes do Curso de Graduação em Odontologia da Universidade Federal de Santa Catarina.

\section{MATERIAL E MÉTODO}

O presente estudo foi realizado após sua plena aprovação pelo Comitê de Ética em Pesquisa com Seres Humanos da Universidade do Estado de Santa Catarina, em dezembro de 2014, sob o CAEE 38390914.3.0000.0118 e sob o parecer de $n$. 917.669.

Classifica-se como uma pesquisa descritiva do tipo transversal, realizada em abril de 2015. Sua população foi composta pelos alunos regularmente matriculados no Curso de Graduação em Odontologia da Universidade Federal de Santa Catarina que haviam concluído a disciplina de Clínica I, 
pertencente ao sexto semestre do curso.

Os dados foram coletados nas salas de aula, após autorização do professor responsável, onde apresentaram-se os objetivos da pesquisa, riscos e benefícios, juntamente com a explicação dos questionários, seguindo-se a distribuição do Termo de Consentimento Livre e Esclarecido.

Os alunos que concordaram em participar do estudo responderam a um instrumento de coleta de dados composto pelo Questionário Sociodemográfico, pelo Questionário da Classificação Econômica Brasil, instituído pela Associação Brasileira de Empresas em Pesquisa ${ }^{11}$, e pelo Teste do Perfil Empreendedor, desenvolvido por Santos ${ }^{12}$ e adaptado para este estudo.

O Teste do Perfil Empreendedor fornece ao participante 15 afirmações, avaliando as respostas através de uma pontuação para quatro alternativas (Discordo totalmente $=1$; Discordo Parcialmente $=2$; Concordo Parcialmente $=3 ; \quad$ Concordo Totalmente=4). Assim, uma somatória dos pontos das 15 afirmações é realizada, resultando em uma classificação final em três possíveis grupos:

a) Perfil empreendedor alto - acima de 50 pontos. É aquele que reúne as características necessárias para realizar um empreendimento de sucesso, já possuindo um conjunto de conhecimento e habilidades pessoais e profissionais de alta qualidade.

b) Perfil empreendedor médio - entre 35 e 50 pontos. Possui um bom potencial, ainda não suficiente para se lançar em um novo empreendimento, necessitando então de aprimoramento para diminuir o risco de fracasso.

c) Perfil empreendedor baixo - Menos que 35 pontos. Necessita desenvolver um esforço adicional para atingir um aprimoramento profissional antes de arriscar empreender.

Após o período de coleta, os dados foram transcritos e tabulados em planilha Excel ${ }^{\circledR} \quad$ (Microsoft Co., Redmond, Washington, EUA) e então submetidos a uma análise estatística descritiva.

\section{RESULTADOS}

De aproximadamente 160 estudantes que haviam concluído a disciplina de Clínica I até o primeiro semestre de 2014, 94 $(58,75 \%)$ disponibilizaram-se a participar da pesquisa e compuseram a amostra final deste estudo. O sexo feminino foi predominante, representando 72,34\% dos participantes.

Em relação à Classificação Econômica, que considera escolaridade dos pais, condições de moradia e posse, metade dos participantes $(50,00 \%)$ foram classificados como classe $\mathrm{A}$, enquanto nenhum estudante pertencia às classes $\mathrm{D}$ ou $\mathrm{E}$. A classe B2 apresentou como a segunda mais frequente, com 22,34\% dos participantes, seguida da classe C1 $(13,83 \%)$, B1 $(11,7 \%)$ e C2 (2,13\%).

Em relação ao Perfil Empreendedor, observou-se uma predominância do perfil médio, presente em $58,51 \%$ dos participantes. O perfil alto, por sua vez, foi observado em $41,49 \%$ dos estudantes. Nenhum dos participantes desta pesquisa enquadrou-se na classificação de um baixo perfil empreendedor.

Entre ambos os sexos, o perfil empreendedor médio foi mais prevalente, sendo $55,9 \%$ entre as mulheres e $65,4 \%$ entre os homens. Entretanto, entre os indivíduos com a tendência alta, $76,92 \%$ foram do sexo feminino.

A distribuição do perfil empreendedor entre as diferentes classes socioeconômicas observadas neste estudo pode ser observada através da tabela 1. 
Tabela 1. Distribuição das frequências absoluta (n) e relativa (\%) do perfil empreendedor entre os estudantes do Curso de Graduação em Odontologia da UFSC conforme a sua classificação econômica.

\begin{tabular}{c|cccccc}
\hline \multirow{2}{*}{$\begin{array}{c}\text { Classificação } \\
\text { Econômica }\end{array}$} & \multicolumn{2}{|c}{ Alto } & \multicolumn{2}{c}{ Médio } & \multicolumn{2}{c}{ Total } \\
\hline A1 & 22 & 46,81 & 25 & 53,19 & 47 & 50,00 \\
B1 & 3 & 27,27 & 8 & 72,73 & 11 & 11,70 \\
B2 & 7 & 33,33 & 14 & 66,67 & 21 & 22,34 \\
C1 & 6 & 46,15 & 7 & 53,85 & 13 & 13,83 \\
C2 & 1 & 50,00 & 1 & 50,00 & 2 & 02,13 \\
Total & 39 & 41,49 & 55 & 58,51 & 94 & 100,00 \\
\hline
\end{tabular}

\section{DISCUSSÃO}

O conhecimento empreendedor, frente ao atual mercado de trabalho, faz-se fundamental. Os acadêmicos de Odontologia devem, ainda na função de estudantes, aprimorar seu aprendizado sobre empreendedorismo bem como seu perfil empreendedor ${ }^{8}$. As habilidades do ser empreendedor não se limitam apenas a gerenciar ou a ser dono de uma empresa privada, mas estão relacionadas à capacidade de liderança e de iniciativa, através do sujeito inovador. A atividade inovadora envolve o trabalho com as situações desconhecidas ${ }^{13}$.

As Diretrizes Curriculares Nacionais são claras ao orientar a formação para o desenvolvimento de habilidades como a liderança, a administração, o gerenciamento e a comunicação9. O profissional deve estar apto para a vida empreendedora, portanto. Observamos nesta pesquisa que os alunos do Curso de Graduação em Odontologia da UFSC apresentam predominantemente tendência média à vida empreendedora, existindo ainda uma fragilidade na formação deste perfil que exige maior aprimoramento.
A própria multidisciplinaridade pertence aos princípios do empreendedorismo. Os empreendedores de maneira geral sabem que o êxito e o sucesso dependem de uma equipe competente, motivada para implantar ideias e desafios ${ }^{4}$.

O papel da universidade neste aspecto deve ser, portanto, voltado para possibilitar o aprimoramento e a transição de uma tendência média para uma tendência alta, preparando assim seus estudantes para a vida profissional.

A prevalência feminina no Curso de Graduação em Odontologia não é exclusividade da Universidade Federal de Santa Catarina, sendo observada também em todo o Brasil. Na Universidade Estadual de Montes Claros, o sexo feminino foi o mais prevalente desde $1996^{14}$. A Universidade Federal do Rio Grande do Sul, por sua vez, tem aproximadamente $69,20 \%$ de estudantes do sexo feminino ${ }^{15}$.

Neste estudo, além da predominância feminina, destaca-se também a maior prevalência do perfil empreendedor alto entre estas, quando em comparação ao sexo 
masculino. Essa diferença também é observada em todo o mercado de trabalho brasileiro, de forma geral, onde 53\% dos 18,8 milhões de empreendedores em estágio inicial são mulheres ${ }^{16}$. O Brasil é considerado como um país de alto empreendedorismo feminino inicial, sendo o $15^{\circ}$ no ranking mundial ${ }^{17}$.

A influência da classe econômica na questão do empreendedorismo também é relatada na literatura. No Brasil, o empreendedorismo em estágio inicial costuma ser menor entre pessoas com mais do que nove salários mínimos, sendo mais frequente entre os grupos com menor renda, mas com pouca variação entre os grupos ${ }^{17}$. Esta pesquisa, embora tenha optado por outro método de classificação, demonstra também que de forma geral, há pouca variação entre as tendências ao empreendedorismo entre as classes.

\section{CONCLUSÃO}

Apesar da importância do empreendedorismo para uma boa formação acadêmica e vida profissional, observou-se que a maioria dos alunos ainda apresenta uma tendência média para seu perfil empreendedor entre os alunos do Curso de Graduação em Odontologia da UFSC, embora uma tendência baixa não foi observada nesta pesquisa.

A tendência empreendedora entre as mulheres é um aspecto destacado de forma geral no estudo do empreendedorismo, também observado nesta pesquisa, onde as mulheres apresentaram maior prevalência da tendência alta para o perfil empreendedor quando comparadas ao sexo masculino.

Fatores como características econômicas não apresentaram diferenças para o perfil empreendedor aqui avaliado. Destacase que, dada a importância do empreendedorismo para a formação em Odontologia, e considerado as Diretrizes Curriculares Nacionais, o estímulo do desenvolvimento do empreendedorismo entre os acadêmicos de Odontologia deve ser estimulado pelas universidades, sendo esta uma importante área de pesquisa e formação.

\section{ABSTRACT \\ Entrepreneurial profile among Dental Students from Federal University of Santa Catarina}

The purpose of this cross sectional study was to assess the Entrepreneurial Profile among undergraduate dental students at Federal University of Santa Catarina. The study used the Sociodemographic Questionnaire, the Brazilian Economic Classification Questionnaire and the Entrepreneurial Profile Test, that were distributed among dental students who have already completed one semester of clinical activity. The Entrepreneurial Profile Test uses a fouroption question method, and uses score to classify students in high, medium or low Entrepreneurial Profile. A descriptive statistical analysis was conducted. Among the 94 students that agreed to participate in this study, majority was women $(73.34 \%)$. The economic classes AI (50.00\%) and BII $(22.34 \%)$. None of the participants were from Class D or E. The Medium Entrepreneurial Profile was observed in 58,51\% of the students, compared to $41.49 \%$ with high Profile. The low Entrepreneurial Profile was not present among the participants. Among high Entrepreneurial Profile students, 76.92\% were women. Class CII presented $50.00 \%$ of high Entrepreneurial Profile, but was composed by just two students. Among Class A1 this value was $46.81 \%$ and $46.15 \%$ for Class C1. This research presented the Entrepreneurial Profile among undergraduate dental students from Federal University of Santa Catarina, where although low Entrepreneurial Profile was not observed, most of students presented medium Profile, requiring more improvements. There is also an emphasis on the entrepreneurial trend among women.

Descriptors: Students, Dental, Innovation. Entrepreneurship. Organization and Administration. 


\section{REFERÊNCIAS}

1. Conselho Federal de Odontologia. Brasil: Conselho Federal de Odontologia; 2016. [Acesso em: 02/03/2016]. Disponível em: http://cfo.org.br/servicos-e-consultas/fa culdades

2. Muylder CF, Lafalce JL, Pires AM. Influência do perfil empreendedor na gestão de uma instituição de ensino. AOS. 2013; 2(2):7-18.

3. Pereira GDF, Cordeiro AT, Pires da Silva MA, Batista MM. Empreendedorismo regional: um olhar sobre a identidade cultural em narrativas locais. Rev Neg. 2013; 18(2):3-26.

4. Galeano R, Meurer V, Previdelli JJ. Empreendendo com Saúde: estudo exploratório do perfil do empresário da área da saúde. RA Unimep. 2005; 3(1):326.

a. Tatto FR, lagemann M, Canever MD. O interesse em tornar-se empreendedor muda com o passar da experiência universitária? XVII Congresso de Iniciação Científica e X Encontro de PósGraduação. 2008; Pelotas. [Acesso em: 02/03/2016]. Disponível em: ufpel.edu.br/ cic/2008/cd/pages/pdf/SA/SA_00874.pdf

5. Ribas MA, Siqueira ES, Binotto Erlaine. O desafio da gestão para profissionais da Odontologia. Encontro Nacional de Engenharia de Produção; 2010(1-13); São Carlos.

6. Santos SC, Caetano A, Curral L. Atitude dos estudantes universitários face ao empreendedorismo: Como identificar o potencial empreendedor? $\mathrm{Rev} \mathrm{Pt} \mathrm{Br}$ Gestão. 2010; 9(4):2-14.

7. Rocha ELC, Freitas AAF. Avaliação do ensino de empreendedorismo entre estudantes universitários por meio do Perfil Empreendedor. Rev Adm Contemp. 2014; 18(4):465-86.

8. Brasil. Resolução CNE/CES 3/2002.
Diário Oficial [da] República Federativa do Brasil, 4 de março de 2002. Seção 1, p. 10 .

9. Ceccon MF. A odontologia em prova. Rev APCD. 2000; 54(5):353.

10. Associação Brasileira de Empresas de Pesquisa. Critério de Classificação Econômica do Brasil; 2014; São Paulo. [Acesso em: 02/03/2016]. Disponível em: http://www.abep.org/criterio-brasil

11. Santos M. Teste do Perfil empreendedor. 2014; São Paulo. [Acesso em: 02/03/ 2016]. Disponível em: mcon.com.br

12. Gomes AF, Lima JB, Cappelle MCA. Do empreendedorismo à noção de ações empreendedoras: Reflexões Teóricas. Alcance. 2013; 20(2):203-20.

13. Costa SM, Durães SJA, Abreu MHNG. Feminização do Curso de Odontologia da Universidade Estadual de Montes Claros. Ciênc Saud Coletiva. 2010; 15(sup1) :1865-73.

14. Amorim RO, Batista LE. Empreendedorismo feminino: razão do empreendimento. Pitágoras. 2012; 3(3): 1-13.

15. Rezende FP, Nakanishi FC, Machado ACP, Quirino MRS, Anbinder AL. Perfil, motivações e expectativas dos graduandos e graduados em Odontologia. Rev Odonto UNICID. 2007; 19 (20):165-72.

16. Global Enterpreneurship Monitor. Empreendedorismo no Brasil. 2012; Curitiba; Gem: 165. [Acesso em: 02/03/2016]. Disponível em: obser vatorio.sebraego.com.br/midias/downloa ds/22072013153931.pdf.

Correspondência para:

Cláudio José Amante

e-mail: claudiojosea@yahho.com.br

Av. Itamarati, 380/304

88034-400, Florianópolis/SC 